\title{
Effects of ketamine on EEG data: a case report
}

Nunes R.R. ${ }^{1}$, Lopes C.G. ${ }^{2}$, Cavalcante S.L.F. ${ }^{1}$, Fernandes M.B.C. ${ }^{1}$, Nunes Filho R.R. ${ }^{1}$, Rodrigues P.V.T. ${ }^{1}$

${ }^{1}$ Hospital Geral de Fortaleza, Dept of Anaesthesiology, Fortaleza, Brazil, ${ }^{2}$ Hospital Sao Carlos, Dept of Anaesthesiology, Fortaleza, Brazil

Background: Ketamine is an arylcyclohexylamine derivative with sedative properties, which is also used in the management of perioperative and neuropathic pain. The compound produces a dissociative state apparently through electrophysiological inhibition of thalamocortical connections and stimulation of the limbic system. In this report we describe an EEG-derived spectrographic pattern observed after ketamine administration.

Case report: A 30-year-old male patient $(80 \mathrm{~kg})$ presenting with acute abdomen from a bullet wound was submitted to general anesthesia (sufentanil $75 \mu \mathrm{g}$, propofol $200 \mathrm{mg}$, succinylcholine $70 \mathrm{mg}$ ), orotracheal intubation and neuromuscular blockade (rocuronium 1ED95\%). Anesthesia was maintained with desflurane adjusted to maintain a BIS value of 45-60 and zero burst suppression. The spectrogram evidenced hypersynchronization of alpha, delta and slow oscillations, with low amplitudes at high frequencies. After anesthesia stabilization, ketamine $(80 \mathrm{mg}$ ) was administered, resulting in a rise in the BIS value from 46 to 68 , a rise in the raw EEG frequency, and high amplitudes predominantly in the beta band, with attenuation of alpha, delta and slow oscillations.
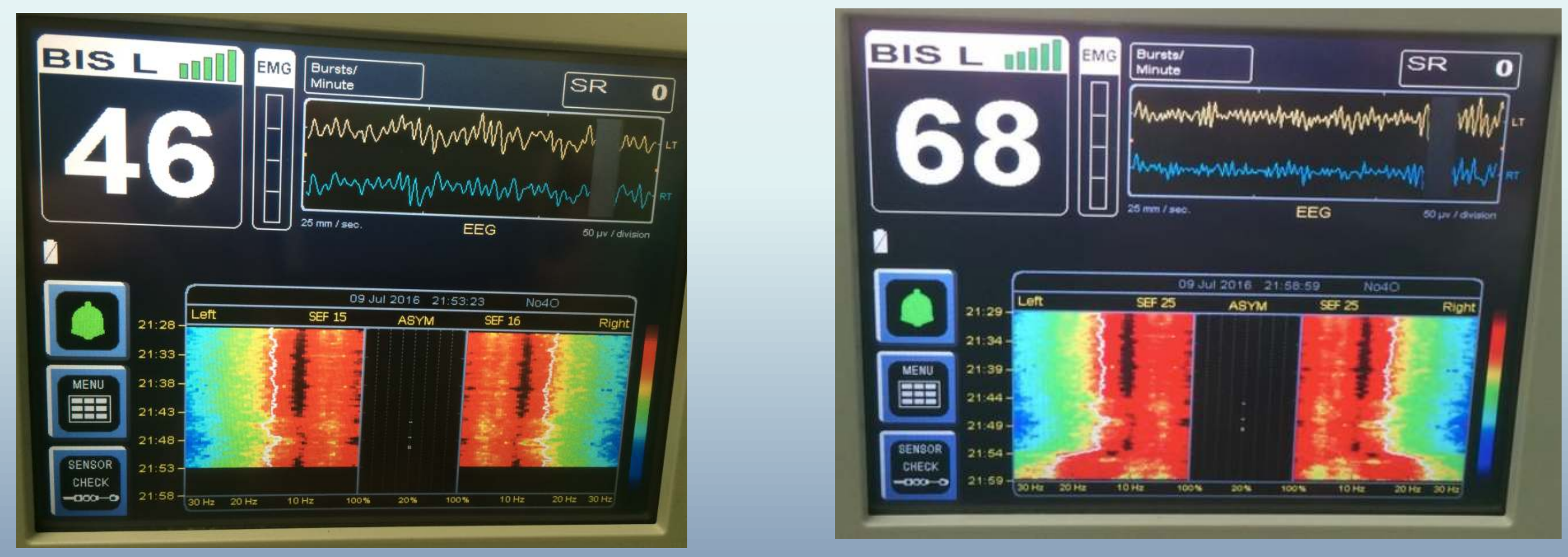

Discussion: Ketamine administration significantly increased beta oscillations and reduced alpha, delta and slow oscillations as a result of the inhibition of the glutamatergic stimulus in the inhibitory GABAergic interneurons, producing aberrant excitatory activity in the cortex, hippocampus and the limbic system. 